\title{
Inclusion body myositis: a case with associated collagen vascular disease responding to treatment
}

\author{
RJM LANE, JJ FULTHORPE, P HUDGSON \\ From the Regional Neurological Centre, Newcastle General Hospital, Newcastle-upon-Tyne, UK
}

SUMMARY Patients with inclusion body myositis demonstrate characteristic histological and electronmicroscopical abnormalities in muscle and are generally considered refractory to treatment. A patient with inclusion body myositis is described with evidence of associated autoimmune disease, who responded to steroids.

The diagnosis of inclusion body myositis depends ultimately on the demonstration of the characteristic intracytoplasmic and intranuclear filamentous inclusions, and cytoplasmic vacuoles originally described by Chou in 1968.' However, reviews of reported cases have also emphasised clinical features which appear to distinguish inclusion body myositis from other forms of polymyositis. ${ }^{2-7}$ Prominent among these are the lack of associated skin changes or other stigmata or collagen vascular disease and failure to respond to steroids.

We report a patient who, while showing the histological and electronmicroscopic features of inclusion body myositis had the typical rash of dermatomyositis, an autoimmune diathesis with pulmonary involvement, and unequivocal clinical improvement in response to steroids.

\section{Case report}

DC (X23980), a 53-year-old man, was referred in May 1977 with a six month history of dyspnoea on exertion, a purple discolouration of the skin over the dorsum of his fingers and hands, arthralgia involving the metacarpophalangeal joints of the right hand, and Raynaud's phenomenon. He denied dysphagia but had lost $4-5 \mathrm{~kg}$ in weight over this period. He had also become aware of considerable wasting of his upper arms and shoulder-girdle

Address for reprint requests: Dr RJM Lane, Regional Neurological Centre, Newcastle General Hospital, Westgate Road, Newcastle upon Tyne, NE4 6BE, UK.

Received 23 March 1984 and in revised form 15 August 1984. Accepted 18 August 1984. muscles. He felt that his legs were quite normal. He denied any sensory symptoms. There was no relevant family or drug history.

On examination, he had a prominent bluish/purple discolouration of the knuckles, thickening of the skin on then dorsum of the hands and a slight heliotrope facial rash. The facial muscles were slightly wasted and he had markedo weakness and wasting of the sternomastoids, deltoids? spinatti, biceps and triceps, with relative preservation of distal muscles. All upper limb reflexes were grosslyg diminished or absent. In contrast, the bulk, power and reflexes in the lower limbs were entirely normal. All modô alities of sensation were intact throughout.

The erythrocyte sedimentation rate was consistently normal $(10-12 \mathrm{~mm} / \mathrm{hr})$ as were the serum muscle enzyme levels (CK 33.5 u/l-normal $<50$; aldolase 1.4 -normal $<5$ ). Autoantibody screen showed positive immunofluorescence to thyroid cytoplasmic antigen but was negative to gastric parietal cell, nuclear, mitochondrial and smooth muscle antigens. The Rose-Waaler test was negative.

A chest radiograph showed reticular and micronodular shadowing in both lungs, particularly at the bases. Vital capacity was 3.11 ( $82 \%$ of predicted value), $\mathrm{FEV}_{1} / \mathrm{FVC}$ was 0.63 (normal >0.75) and single-breath CO transfer factor was reduced to $33 \%$ of expected value, at 3.11 $\mathrm{mmol} \mathrm{min} \mathrm{m}^{-1} \mathrm{kPa}^{-1}$. The arterial blood gases were normal at rest. The pulmonary function tests were considered consistent with the chest radiograph findings, indicating diffuse interstitial pulmonary fibrosis.

Motor and sensory nerve conduction velocity measurements were normal throughout. Concentric needle electromyograms of the right biceps, triceps, quadriceps and tibialis anterior showed no spontaneous activity, and volitional activity was also normal (Dr DD Barwick).

Muscle biopsies were obtained from the left quadriceps and left deltoid. Muscle from the quadriceps was virtually $\sigma$ normal, apart from moderate, non-specific Type 2 fibre $N$ 
(a)

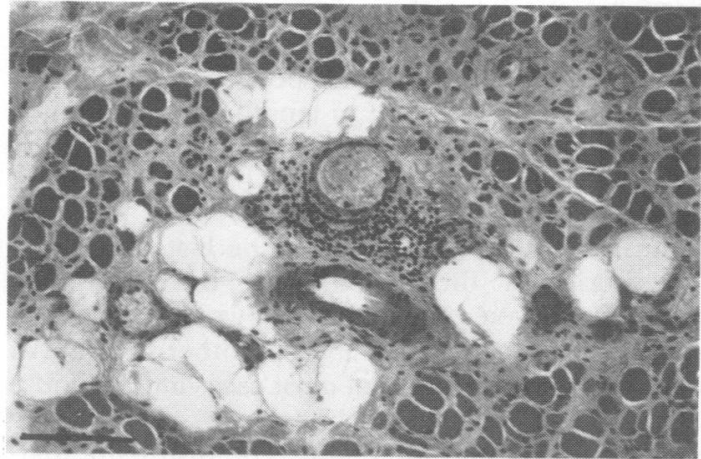

(b)

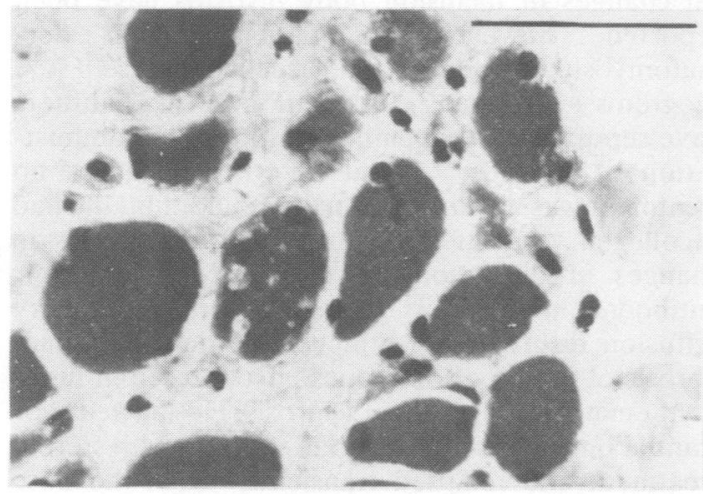

(c)

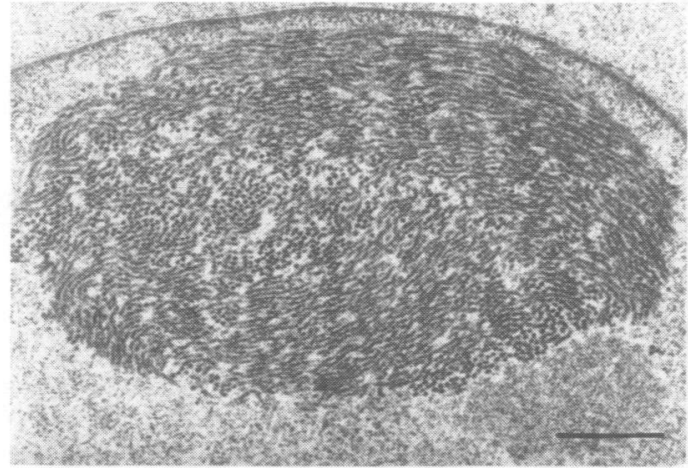

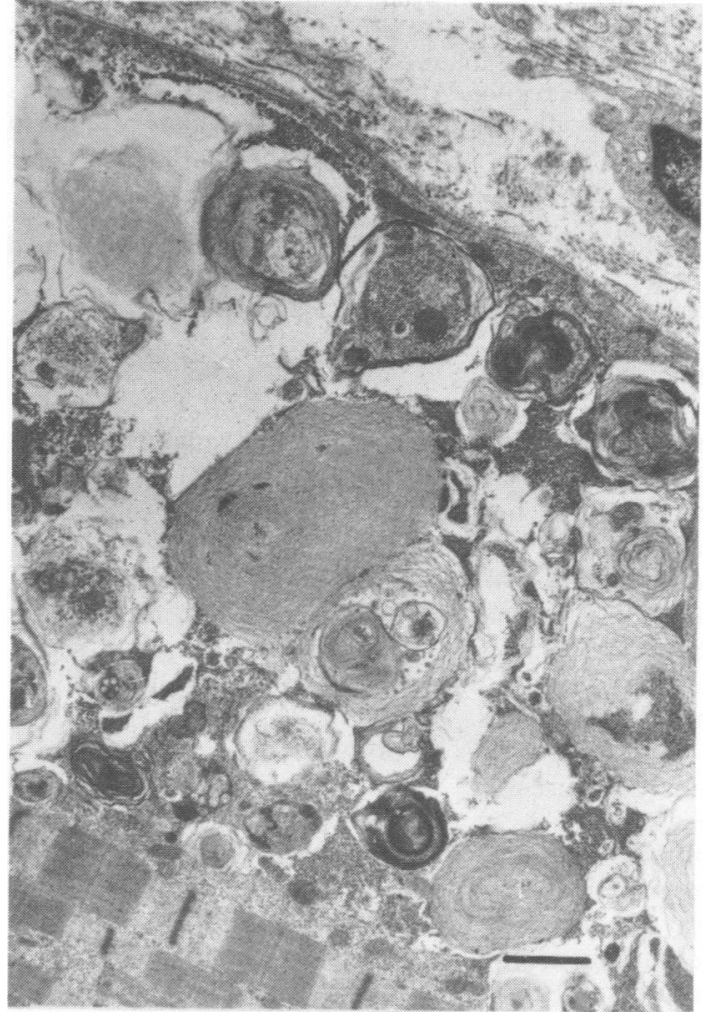

(d)

Fig (a) Section from left deltoid showing perivascular and interstitial inflammatory cell infiltration, marked increase in variation in fibre diameters and replacement of muscle with fat and fibrous tissue. ( $H \& E .100 \mu$ marker). (b) Representative area from (a) showing sub-sarcolemmal vacuoles with basophilic inclusions (arrows). ( $H \& E .50 \mu$ marker). (c) Intranuclear filaments in a nearby nucleus. ( $0.5 \mu$ marker). (d) Membranous whorls forming aggregates, corresponding to the basophilic masses in a sub-sarcolemmal vacuole. (1 $\mu$ marker).

atrophy. However, the deltoid biopsy specimen showed marked fibrosis with striking perivascular and endomysial lymphocytic infiltrations, and gross variation in muscle fibre diameters (fig (a)). Some of the atrophic fibres were angulated, but there was no evidence of fibre type grouping or grouped fibre atrophy on histochemical stains. Under high power, some $5 \%$ of the fibres showed conspicuous vacuoles, some containing basophilic masses at their peripheries (fig (b)). These could be seen in more detail in the toluidine blue-stained sections prepared for electronmicroscopy. Electronmicroscopic examination revealed the presence of both cytoplasmic and intranuclear (fig (c))

filaments in random orientation. The filaments measured $20 \mathrm{~nm}$ in diameter. In addition, numerous osmiophilic membranous whorls were observed (fig (d)), corresponding to the basophilic masses in the vacuoles seen by light microscopy.

The patient was treated with prednisone $20 \mathrm{mg}$ tds and azathioprine $50 \mathrm{mg}$ tds. On review one month later, he felt much better and his strength had improved. Over the succeeding month his skin rash gradually faded and the steroids were reduced to $30 \mathrm{mg}$ daily. This resulted in a return of his symptoms and progressive exertional dyspnoea. Reinstitution of prednisone $20 \mathrm{mg}$ tds again pro- 
duced significant improvement to his arm and shoulder strength and reduction of his dyspnoea. Further improvement in breathing followed addition of a beclomethasone inhaler to his regime. Attempts at steroids reduction some five months after starting treatment once more resulted in deterioration, particularly in his breathing but his condition had stabilised after six months of treatment on highdose steroids.

Unfortunately, in February 1978 he suddenly developed a right hemiplegia and dysphasia and eventually succumbed to a suppurative bronchopneumonia.

\section{Discussion}

Since Chou originally described "myxovirus-like structures and accompanying nuclear changes in a patient with chronic polymyositis", inclusion body myositis has emerged as an apparently distinct pathological entity. Furthermore, the clinical features in the majority of more than forty cases reported to date, have conformed to a pattern distinct from that normally encountered in autoimmune polymositis. ${ }^{2-7}$

Typically, inclusion body myositis presents with slowly progressive, sometimes asymmetrical, painless weakness which often affects the distal limb muscles as much, or even more prominently, than the proximal. Facial muscle weakness and dysphagia may be early manifestations. There has been some debate regarding the sex ratio in inclusion body myositis. Reviews of reported cases revealed a considerable male predominance, contrasting with the female predominance in autoimmune myositis. ${ }^{34}$ The condition tends to affect an older population than autoimmune polymyositis, although it may also occur in younger patients, who are more often female, and a bimodal age-sex distribution has been suggested. ${ }^{6}$ Serum muscle enzymes are generally normal, or only moderately raised.

Electromyography often reveals predominant changes of denervation with fibrillations and large prolonged duration polyphasic potentials with late components on volitional activity, but in some cases the changes may be predominantly myopathic.

Muscle biopsy findings in inclusion body myositis are said to be diagnostic. ${ }^{2-7}$ By light microscopy, $5-10 \%$ of the fibres reveal single or multiple membrane-lined vacuoles, often containing basophilic masses, and occasionally eosinophilic inclusions. The vacuoles are typically acid phosphatase negative, indicating that they are probably not autophagic. In addition to necrosis, phagocytosis and replacement of muscle by fat and fibrous tissue in chronic cases, most cases of inclusion body myositis reveal lymphocytic inflammatory cell infiltrates, although these may be sparse or even absent in some cases. The electronmicroscopic appear- ances, however, are characteristic. There are cytoplasmic and intranuclear masses of randomly orientated filaments, reported to be between $10-25 \mathrm{~nm}$ in diameter and about $1-3 \mu$ in length, and membranous osmiophilic whorls within the vacuoles, which are believed to correspond to the basophilic masses seen by light microscopy.

Our patient thus conforms to the histopathological criteria for the diagnosis of inclusion body myositis. However, previous reviews have emphasised the absence of skin changes or other stigmata of collagen vascular disease in most cases and response to treatment in inclusion body myositis has been almost uniformly unsuccessful. The histopathological changes of inclusion body myositis have been reported, however, in patients with dermatomyositis ${ }^{89}$ scleroderma, $^{10}$ coeliac disease, ${ }^{11}$ and Sjogren's syndrome..$^{2}$ In addition several authors have reported modest clinical response to administration of steroids, ${ }^{4511}$ but in other instances no treatment was given. ${ }^{34}$ Our patient undoubtedly had a collagen vascular disorder, with the typical skin changes of dermatomyositis, thyroid cytoplasmic antibodies and a chest radiograph and pulmonary diffusion disorder indicating interstitial pulmonary fibrosis of the type sometimes encountered in lupus erythematosus or systemic sclerosis. He showed substantial improvement in muscle strength with steroid treatment and relapsed transiently when the dose was decreased.

Clearly therefore, this case calls into question the status of inclusion body myositis as a separate nosological entity. It also emphasises the importance of at least attempting therapy in this condition.

We thank Dr C Strang for allowing us to report this case. Dr Lane is a Wellcome Senior Research Fellow in Clinical Sciences.

\section{References}

' Chou SM. Myxovirus-like structures and accompanying nuclear changes in chronic polymyositis. Arch Pathol 1968;86:649-58.

${ }^{2}$ Carpenter S, Karpati G, Heller I, Eisen A. Inclusion body myositis: A distinct variety of idopathic inflammatory myopathy. Neurology (Minneap) 1978;28:8-17.

${ }^{3}$ Danon MJ, Reyes MG, Perurena OH, Masden JC, Manaligod JR. Inclusion-body myositis. A corticosteroidresistant idiopathic inflammatory myopathy. Arch Neurol 1982;39:760-4.

4 Julien J, Vital CL, Vallat JM, Lagueny A, Sapina D. Inclusion body myositis. Clinical biological and ultrastructural study. J Neurol Sci 1982;55:15-24.

${ }^{5}$ Mikol J, Felten-Papaiconomou A, Ferchal F, et al. Inclusion-body myositis: Clinicopathological studies and isolation of an adenovirus Type 2 from muscle 
biopsy specimen. Ann Neurol 1982;55:15-24.

- Sawchak JA, Kula RW, Sher JH, Shafig SA, Clark LM. Clinicopathologic investigations in patients with inclusion body myositis. Neurology (NY) 1983;33: (suppl 2), 237: (abs.)

${ }^{7}$ Eisen A, Berry K, Gibson G. Inclusion body myositis (IBM): Myopathy or neuropathy? Neurology (NY) 1983;33: 1109-114.

${ }^{8}$ Chou JM. (1972). Prospects of viral etiology in polymyositis. In: BA Kakulas, ed. 2nd International Congress on Muscle Diseases Part 2. Amsterdam: Excerpta Medica, 17-28.
${ }^{9}$ Sato T, Tsubaki T. Characteristics of virus-like structures in polymyositis. ibid, 280-283.

${ }^{10}$ Marie L. (1980) Contribution à l'Étude d'une Nouvelle Entité Clinico-pathologique: la myosite à inclusions-à propos d'une Observation associée à une Sclérodermie Généralisée, un Syndrome de Kleinefelter. Thèse, Paris-Sud.

" Hughes JT, Esiri MM. Ultrastructural studies in human polymyositis. J Neurol Sci 1975;25:347-60.

12 Chad D, Good P, Adelman L, Bradley WG, Mills J. Inclusion body myositis associated with Sjögren's syndrome. Arch Neurol 1982;39:186-8. 